\title{
Integrating Atomic Force Microscopy for Simultaneous Investigations
}

\author{
Helen A. McNally* and Dominic D’Agostino** \\ * Departments of Electrical and Computer Engineering Technology and The Birck Nanotechnology \\ Center, Purdue University, West Lafayette, IN 47907 \\ **Department of Molecular Pharmacology and Physiology, University of South Florida, Tampa, Fl \\ 33612
}

Since its inception in 1986 [1], Atomic Force Microscopy (AFM) has become a standard technique in the microfabrication industry, academic departments such as materials science, chemistry, and biology, and even for field maneuvers performed by the military. Investigation of sample topography and material characteristics at the nanoscale are the primary uses of this instrument. The AFM can also be used to manipulate the sample of interest. Experiments can be performed in vacuum, air, fluid and hyperbaric conditions making this a most versatile instrument with unparalleled imaging and measurement capabilities. Manufacturers have been integrating typical optical microscopes with AFM for the past five years allowing standard. However, researchers are beginning to integrate AFM with additional measurement techniques to increase the utility of AFM and allow simultaneous analysis by a variety of systems. This paper will discuss recent integrations of AFM and provide examples of combined data sets collected.

Optical techniques were the first to be integrated with AFM as it has become of particular importance to the biological community [2.] The stage of standard optical microscopes is slightly modified to accommodate an AFM allowing techniques such as bright field, phase contrast and differential interference contrast to be easily combined with AFM data. An integrated optical microscope/AFM is shown in Fig. 1. Fluorescence microscopy can also be performed on the same platform. A combined data set of fluorescence and AFM data is shown in Fig. 2. More complex systems such as confocal microscopy and multiphoton microscopy can also be integrated into the combined optical/AFM system. Software packages such as Bruker's Microscope Image Registration and Overlay (MIRO ${ }^{\mathrm{TM}}$ ) have been developed to overlay these data sets and increase the data analysis capable of each system individually. Of course these integrations do come with a price. Generally the noise associated with the larger optical microscope platform and the moving parts of a spinning confocal system will decrease the resolution limits of the AFM.

Combining AFM with chemical analysis techniques has recently become of interest. The AFM is capable of some chemical analysis alone by modifying the tip for selective interaction with the sample. RAMAN spectroscopy has been used for years to evaluate the chemical nature of samples. Recently many AFM manufactures have been modifying their systems to accommodate the necessary components to perform simultaneous AFM and RAMAN spectroscopy. Others have incorporated the AFM electronics into the chamber of a scanning electron microscope (SEM.) In most cases the systems are operated under vacuum conditions which limits the overall capability. However as more SEM environmental chambers are developed it is expected these will also incorporate AFM.

Overall AFM is still a developing technology with extraordinary stand alone capabilities and limitless possibilities for integration with additional techniques. AFM operating conditions, modes 
and tips are being continually being developed and/or modified to investigate samples in new ways. It is expected that additional integrations of AFM will also be developed as researchers dream of new questions and search to examine their samples in unconventional ways.

\section{References}

[1] Binnig, G., Quate, C.F., \& Gerber, Ch., Atomic Force Microscopy, Physics Review Letts, V. 56, N. 9, pp. 930-933., 1986.

[2] Morris, V.J., Kirby, A.R., and Gunning, A.P., Atomic Force Microscopy for Biologist, Imperial College Press, Norwich, UK, pp.254, 1999.

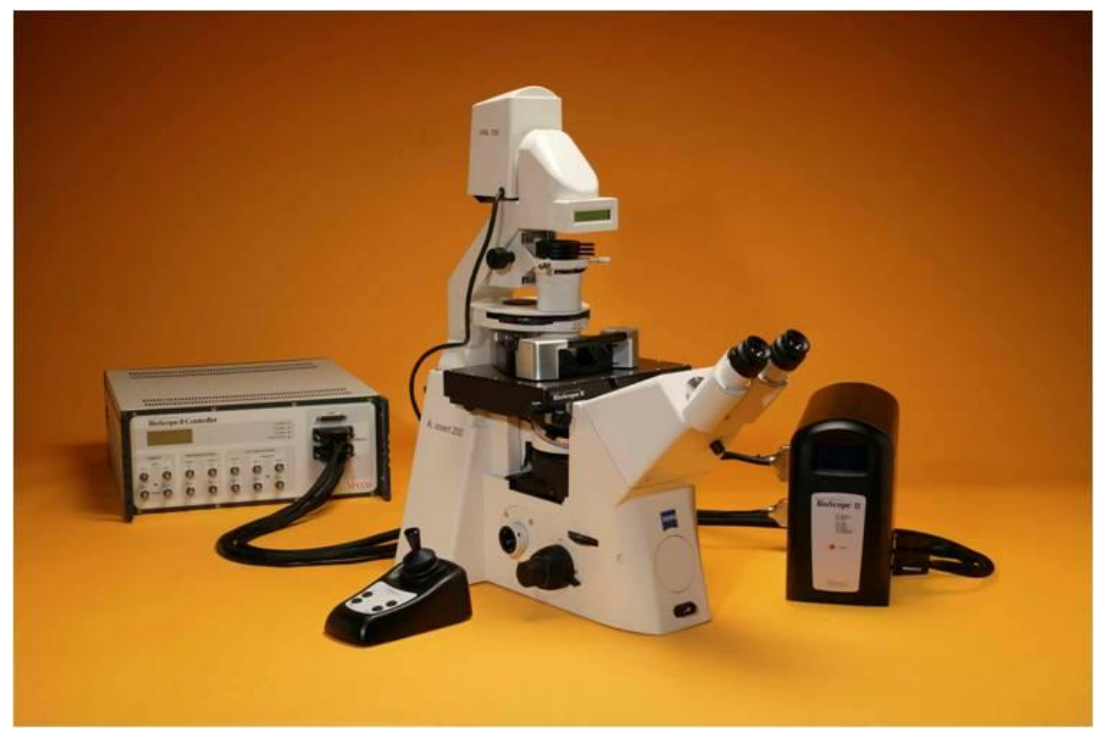

FIG. 1. Bruker Bioscope II Atomic Force Microscope. AFM is mounted on optical microscope stage. Also seen is the associated Electronics box, controlled and joystick.

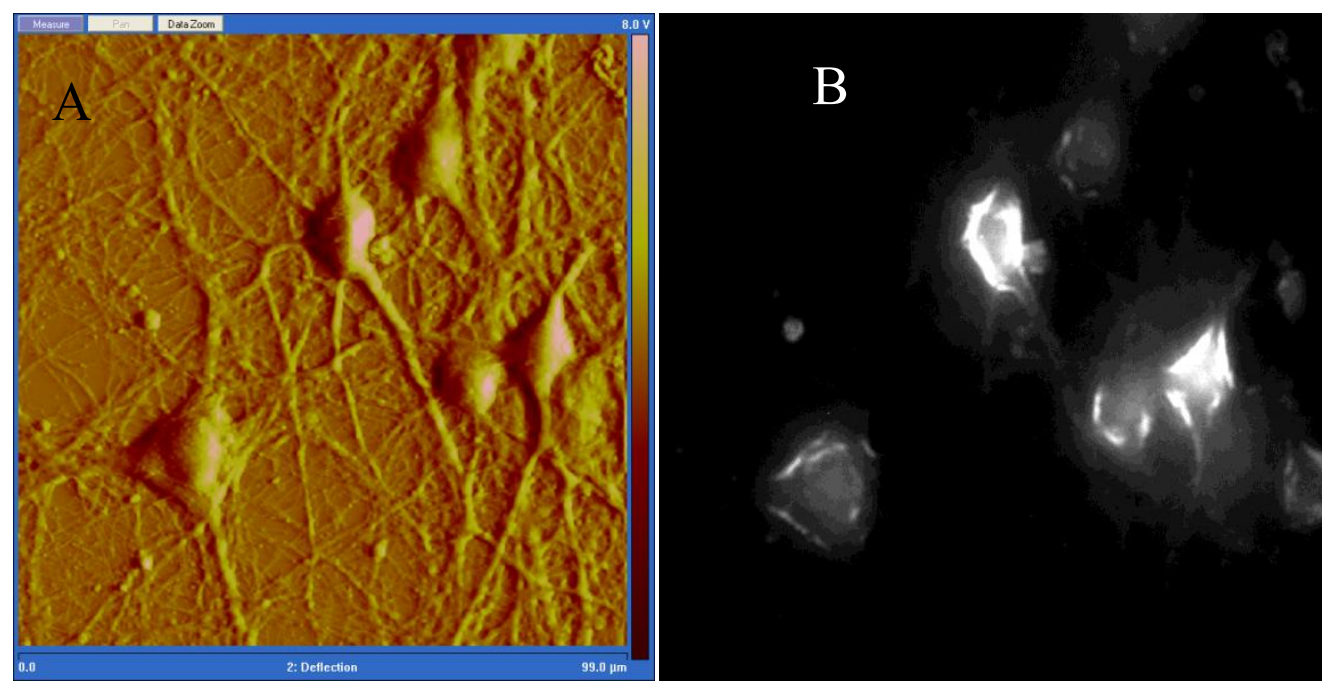

FIG. 2. Combined data set of AFM and fluorescence using a Bioscope SZ. A, AFM scan of primary cortical neurons after chemical hypoxia (azide). B, Fluorescence microscopy on the same neurons was used to assess increased membrane permeability with ethidium homodimer-1 as an early event preceding necrosis. 